\title{
Internet of Things (IOT) as the future of networked devices: An overview
}

\author{
Godson S Olusanya ${ }^{1,}{ }^{*}$, Charles Okunbor ${ }^{2}$ and Oghenetega Avwokuruaye ${ }^{3}$ \\ ${ }^{1}$ Babcock University, Ilishan Remo, Ogun State, Nigeria. \\ ${ }^{2}$ Admiralty University, Ibusa, Delta State, Nigeria. \\ ${ }^{3}$ Department of Cyber Security, Admiralty University, Ibusa, Delta State, Nigeria.
}

Global Journal of Engineering and Technology Advances, 2021, 09(03), 031-037

Publication history: Received on 16 November 2021; revised on 12 December 2021; accepted on 14 December 2021

Article DOI: https://doi.org/10.30574/gjeta.2021.9.3.0154

\begin{abstract}
The internet being a major tool in the globalization of the world has made it possible for human beings to interact, share and access information on a very large scale without distance being a barrier. Computer systems with broad functionalities like the desktop, laptop and smart phone are connected through the internet. But going beyond just connecting computer systems to the internet, the Internet of Things connects devices and objects together and to the internet. The Internet of Things (IoT) is a developing paradigm that makes communication and gathering of data possible between electronic devices, objects and sensors through internet technology, with the aim of improving quality of life. IoT is seen to be a big player in the networking of future devices and the internet but has some challenges that needs to be addressed. This paper through extensive literature review, highlighted the progress made by IoT, its applications in different sectors, the challenges faced by this technology for its full actualization and also its future projections.
\end{abstract}

Keywords: Application; Devices; Internet of things; Internet technology

\section{Introduction}

Internet of Things (IoT) is a technology which bestows intelligence on less intelligent electronic devices and connects them together for information sharing, interaction and feeding of a larger system which will process the information gotten so as to aid more informed decision making [1]. IoT creates a network of connected electronic devices and objects that gather data in the environment through sensing, communicate and share the gathered data, analyze and process data to give information and initiate action based on the information gotten $[2,3,4,5]$.

Computer systems are able to interact and share information using the existing internet structure but devices such as home appliances, objects and things in general, are incapable of accessing the internet without the IoT technology acting as a connecting structure. The IoT technology as it has come to be, creates a link between these devices (or things) making them able to communicate with one another by way of sharing data and serving as devices that feed data into larger systems $[3,5,6]$.

Beyond connecting computer systems like; laptops, desktops and smart phones, researchers hope to connect more and more devices, objects and everyday things around us to the internet through the Internet of Things. What therefore will the Internet of Things deliver to us in the coming years? Research has shown that IoT has a lot of prospects; it can, in a nutshell, deliver an improved quality of life based on its applications. These applications include but not limited to data gathering, processing, interpretation, communication/sharing and these open up a whole new world of challenges for data gathering and management.

\footnotetext{
* Corresponding author: Godson S Olusanya

Babcock University, Ilishan Remo, Ogun State, Nigeria.
} 


\section{Literature review}

In a call made for a common ground (standardization) to be developed for IoT, Bassi and Lange stated that a proper standard if developed will ensure interoperation, heterogeneous connectivity across platforms [7]. This standardization will facilitate a proper understanding of the data transmitted from platform to platform, ensure that the right format of data is stored and guarantee data correctness for processing.

Researchers' works have shown that the Internet of Things has a lot to offer to us. Bhuvaneswari and Porkodi highlighted some of the applications of IoT in their work [1]. They gave society, environment and industry as the broad classification of the areas that IoT will impact. This impact will deliver a display of smartness and connection to the internet for data transmission and processing. However, IoT is experiencing some major challenges that are limiting its full potential actualization some of which include; architecture and standardization, privacy and security, data intelligence or management, among others.

The view point of Wortmann and Fluchter in their evaluation of the value creation of IoT reflects a position that sees beyond what individually connected things can deliver but what an array of connected things can deliver and the value the data gathered from the connected things present [8]. One of which could be the optimization of farm equipment, if these are the connected things, and monitoring for usage and performance. Their work equally attested to the limitations preventing IoT's full actualization.

Satpute and Deora identified data management as one of the limitations of IoT in their work [9]. They believe that the data gathering functionality of IoT has the capacity to accumulate a lot of data both relevant and irrelevant and send same via the internet to the cloud [9]. This process burdens the network and storage capacity therefore, they suggested a local IoT controller unit to limit the volume of data that is transmitted to a central storage location in the cloud. This local IoT controller unit will filter off less relevant data and ensure that relevant and appropriate ones are transmitted.

Miraz, Ali, Excell and Picking opined in their paper that there could be three classifications as regards network of things, objects, devices and even people [10]. The classes are: "Internet of Things" (IoT) which is a network of devices, "Internet of Nano Things" (IoNT) which is a nano-network of nano-sensors and "Internet of Everything" (IoE) which is a network of people, data, process and things. They also identified some of the common limitations surrounding IoT in terms of standardization, energy source, just to mention a few.

Patel and Patel in their work, highlighted certain salient issues concerning IoT: definition, characteristics, architecture, enabling technologies, application and future challenges [4]. The researchers further explained the meaning of IoT, and categorized its enabling technologies into three: those that enable IoT to gather data from its environment, those that enable it to process the data and those that improve security and privacy. In identifying the challenges, privacy and security, cost versus usability, interoperability, data management and energy issues were highlighted.

Atzori, Lera and Morabito analyzed the Internet of Things phenomenon from an evolutionary point of view, by emphasizing that the IoT has undergone several transformations in its characterizing technologies and principles since its introduction [11]. The researchers identified three (3) generations of IoT. The first one was of tagged objects; the second one of things interconnection through web technologies; the third one of social objects, semantic data representation, and cloud of things.

There exists a common understanding that there are limitations which span across data management, standardization, privacy and security, energy sources, infrastructure, among others. It is the belief of Bhuvaneswari and Porkodi along with Patel and Patel $[1,4]$ that due consideration be given to how the gathered data is handled. Moving beyond just accumulating data via sensing ability, we must consider succinctly, what we do with the data and the kind of intelligence we are able to derive from them.

It is no news that the application of IoT technology is around us in this day and age. That which was once envisioned by researchers is becoming a reality today. IoT has been envisioned to connect things, devices and objects that are around us granting us access to them anytime and anywhere that is, we may be using virtually anything with internet connectivity $[1,4,10,12]$ but it is becoming more and more feasible with today's technological advancements. It is rightly stated by Patel and Patel "These interconnected objects have data regularly collected, analyzed and used to initiate action, provide a wealth of intelligence for planning, management and decision making" [4].

The Alliance for Internet of Things Innovation in their paper, discussed various identification needs with related use cases and requirements [13]. They further looked at identifier standards, their applicability for the different identifier 
needs and discussed identifier allocation, registration, resolution, security, privacy and interoperability. The paper provided a structured approach by classification of identifier usage and a categorization of requirements. The researchers admitted that in general, no single identification scheme fits all needs and that many identifications are already standardized and are in use.

Kumar, Tiwari and Zymbler, in a review paper, see IoT as not only providing services but also generating huge amount of data [14]. They stressed the importance of big data analytics to provide accurate decision which could be of help in developing enhanced IoT systems. They also discussed some issues and shortcomings in the present technical approaches [14]. The importance of its application in different domains was also highlighted in this research.

Hassan, Qamar, Hasan, Aman and Ahmed carried out a study that covered the applications of IoT in the areas of healthcare, environmental, commercial, industrial, smart cities, and infrastructural [15]. Their work explained the concept of IoT and identified data privacy and scalability, authorization and cost issues, mobility and architectural problems for smart city applications, cost and implementation difficulties for commercial applications, hardware and production issues for industries, standardization and trust issues as the challenges faced by IoT. Their research proposed a next-generation protocol as a solution to some of the challenges. These researchers also suggested the use of Block chain technology to help achieve extra safety, automatic business management, distributed platforms and offline-to-online information authentication [15].

This research work will be anchored on future projections of IoT and deriving intelligence from data for decision making and strategy optimization. This prognosis for IoT cannot be overemphasized because it is projected that there will be over 100 billion connected IoT devices and a global economic impact of more than $\$ 11$ trillion by 2025 [16, 17].

\section{Applications of IOT}

Bhuvaneswari and Porkodi broadly classified IoT applications under three (3) domains [1]:

- Society: IoT applications concerned with improving, developing the society, cities and people.

- Environment: IoT applications that help to protect, monitor and develop natural resources.

- Industry: IoT applications involving financial and commercial transactions between companies and organizations.

However, IoT is being applied in different sectors in the society and different areas of human existence, some of which are highlighted below:

- Health Care: IoT is being used as wearable devices that are used to gather patients' information and save online for medical practitioners to access in order to determine the medical state of the patients and how to diagnose further. There are also wearable devices that are used by individuals to monitor their health status.

- Emergency Situations: IoT systems equipped with sensors/detectors are able to detect the presence of fire in the environment by identifying the presence of certain features like smoke, rise in temperature and even fire intensity then alert the responsible individuals for further action to be taken. They are also used to detect smoke or gas leakages in factories.

- $\quad$ Agriculture: WSNs can be deployed to help monitor soil moisture, available nutrients and weather conditions so as to get the information required to determine when to wet, apply nutrient supplements and generally take decisions that will help ensure a successful planting and harvesting period. Also, WSNs can be used to track livestock and monitor their movements.

- Security: Perimeter Access Control is a way to curb unauthorized access to certain premises. This system is able to determine when there is a forced entry into secured locations and alert those charged with the responsible to guard the place so they can respond accordingly.

- Factory/Industry: Industrial machine developers like Siemens have been able to develop systems that can connect industrial machines and human control terminals; both those that are locally present on-site and the ones with remote access making it possible for workers to remotely access the machines.

- Smart Home: This is the implementation of automation in homes. This kind of system is able to recognize and interpret spoken instructions that are to be executed. This application of IoT in homes is still partial, not yet 
fully implemented however, companies like Samsung (SmartThings) and Amazon have been able to develop AI software, making it possible for users to control home appliances both online and offline.

- Transportation: IoT application in this area assists with traffic control, real time information dissemination to citizens concerning emergency situations and gridlocks. It also helps with traffic diversions when there is a road block due to repairs, reconstructions, as so on.

- Smart City: This combines IoT applications in different areas such as transportation, smart homes/buildings, agriculture, factories, hospitals, and the likes, to function. Its main goal is to improve the quality of life for the citizens.

- Retail/Marketing: IoT can help with marketing products by giving information about them when buyers move close to them in the stores. They also assist with self-checking out (payment without meeting a human attendant) when they are done with purchasing.

- Military: WSNs and smart dusts can be used to stealthily gather information concerning opposing forces to determine their strategies. They can also be used to man country borders so as to be able to detect unauthorized intrusions.

- Others: Smart Metering, Smart Energy Grids, Entertainment, Culture and Tourism, among others.

\subsection{Some existing products that adopt IOT technology}

- Amazon's Echo Auto: A small hands-free device used in the car as a driver's assistant, is able to provide traffic reports, update online shopping carts, play music via the infotainment system in the car and help control appliances in the home using its connection with the home automation system [18].

- FIXD: A mobile app and device which falls under the data gathering via sensors function of the IoT. The sensor device which is connected to an OBD-II port of a vehicle gathers information about the vehicle and relays it to the mobile app. When there is a vehicle fault, it gives explanation in plain language and guides the owner through a 'Do-It-Yourself' diagnosis and repair [19].

- Car Play from Apple and Android Auto: Car infotainment platforms that link smart phones for us to have access to mobile apps such as online maps and voice activated controls without directly operating the smart phones [20].

- Nokia BPM+: Wireless blood pressure monitor; It is a device that is self-operated and it relays the details concerning the user's blood pressure via a mobile app [21].

\subsection{Challenges faced in IOT'S full actualization}

Internet of Things is working towards full applications in virtually every area of man's existence; transportation and traffic control, education, home automation, smart cities, energy grid, medical, wearable devices, entertainment, and so on. However, this full actualization remains a projection because of the present limiting factors.

Below are some of the limiting factors:

- Standardization: IoT is supposed to be able to connect any device using any platform. However, there is yet to be an acceptable standard for heterogeneous object connectivity.

- Privacy and Security: Making various forms of data available online, makes owners of data vulnerable to attacks from malicious persons.

- Data Management: Storage of data, carrying out proper analysis and determining how to fully maximize the data gathered by sensors is still lagging behind.

- Energy Sources: The devices in use today are battery powered and they require regular swapping but some devices will be used in locations where humans will not be able to regularly swap them.

- Infrastructure: If IoT must deliver connectivity to anything, anywhere and anytime, then researchers must work on the infrastructure that will make this a reality.

\subsection{Data handling}

Data is very important to IoT devices in taking decisions and executing a specific task. Data are gathered by these devices using attached sensors. Over time, this data accumulates in the cloud but they are not fully utilized. Patel and Patel 
strongly opined that there is a wealth of intelligence that can aid planning and decision making in the data that interconnected devices gather [4].

Beyond just analyzing data and interpreting what it says, what can it be used for in terms of improving business output, optimizing operations and processes and ensuring profitability? Heflin's write-up made reference to data analysis and how it can help with business improvement [22]. However, He sees this assertion to be too general. Data gathered over time about a piece of equipment for instance, does not just give us information about the equipment but it tells a story about:

- The suitability of the equipment for certain tasks.

- Its operational capability in certain weather conditions.

- How effectively it performs some tasks over others.

- The correctional decisions that must be taken to get more productivity from it.

Data accumulated through sensors of IoT devices, is a wealth of information to give directions for improvements, explorations, corrections and modifications when properly analyzed and interpreted. Therefore, it is important to go beyond extracting the information gotten through analysis of data to understanding the story it tells about any product and environment so as to take the required decisions towards implementing changes.

\section{Prospects of IOT}

Despite the already identified challenges, IoT still has prospects. Its application in the future will be very beneficial to mankind. It will create ease in system access, management and usage. It will make it possible for human beings to interact with everyday objects and devices around them. Connecting every object, every device, everything and virtually anything to the internet is the goal of the Internet of Things [4]. Bhuvaneswari and Porkodi stated in their work that IoT will bring a future where physical objects will be connected to the internet and identify themselves with other devices/objects [1].

IoT will become an efficient system capable of transmitting, providing and sharing the best possible data feedback on our physical health and the best possible monitoring of real time events that would help in decision making related to day to day events [9]. According to Malik, Magar, Verma, Singh and Sagar, IoT is expected to be the future internet in the upcoming years [23]. The researchers also proposed that the long-term of this technology should be investigated and analyzed as many innovative technologies have their calamitous sides.

IoT which has emerged as the technology for connecting objects and devices together for communication, data sharing and internet access boasts of connecting and will continue to connect more devices than the internet has been able to do. Beyond just creating a network for things, IoT has the ability to connect these things to the existing internet of computers. This, by implication, makes Internet of Things the future of networked devices. Figure 1 shows a network for the current internet of computers, another for the emerging internet of things and an overlap for the network of anything and everything. This diagram is an overview of the nervous system of the future internet.

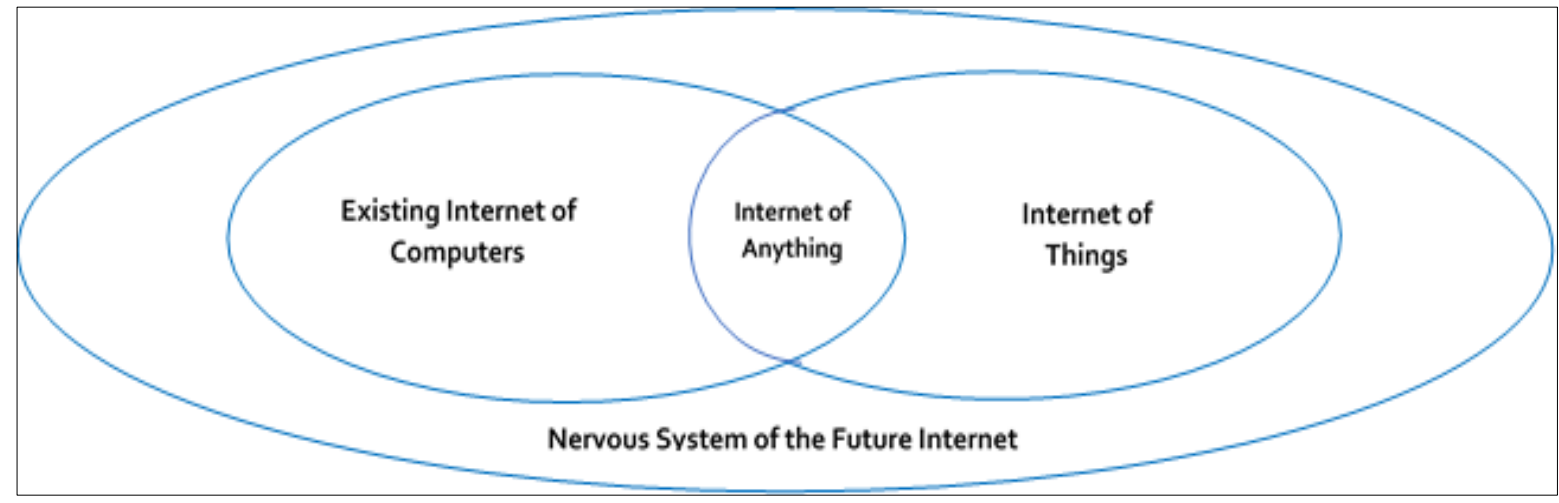

Figure 1 Nervous System of the Future Internet

Above and beyond just connecting devices and things to the internet, the future of IoT holds more real time applications in industries like: automobile, aeronautics, and many others. Also, IoT will look towards providing solutions to existing 
challenges around us. This will be the fulfillment of Gershenfeld's idea of embedding solutions in the everyday objects around us [24].

\section{Conclusion}

Despite the existing challenges, there is confidence that the different scholarly works that are done and will still be done will lead us to the full actualization of the IoT concept. There will be more connections of devices and objects that are yet to be connected at the moment, the realization of smart home through the connection of home gadgets, electrical appliances, smart cities with communication between cars, buildings, filling stations and other objects (moveable and immoveable). In a nutshell, there will be a full realization of Internet of Things - a network of anything with the capability of accessing the internet anywhere, anytime.

\section{Compliance with ethical standards}

\section{Acknowledgments}

The authors also acknowledge themselves for their time, contributions and successful collaboration.

\section{Disclosure of conflict of interest}

The authors do not have any conflict of interest in the writing of this article

\section{References}

[1] Bhuvaneswari V, Porkodi R. The Internet of things (IoT) applications and communication enabling technology standards: An overview. International Conference on Intelligent Computing Applications. 2014; 324-329.

[2] Channel Futures. IoT past and present: The history of IoT, and where it's headed today [Internet]. 2016.

[3] Guillen-Perez A, Cano M-D. Intelligent IoT systems for traffic management: A practical application. IET Intelligent Transport System. 2021; 15: 273-285.

[4] Patel KK, Patel SM. Internet of things - IOT: Definition, characteristics, architecture, enabling technologies, application \& future challenges. International Journal of Engineering Science and Computing. 2016; 6: 61226131.

[5] United Nations Social Enterprise Facility (UNSEF). A brief history of the IoT. Workshop on Internet of Things Development for the Promotion of Information Economy, Boracay, Philippines. 2015.

[6] McFarlane D. The origin of the Internet of things [Internet]. 2015.

[7] Bassi A, Lange S. Chapter 2: The need for a common ground for the IoT: The history and reasoning behind the IoT-A project. In: Bassi A. et al., eds. Enabling Things to Talk. 2013; 13-16.

[8] Wortmann F, Fluchter K. Internet of things - Technology and value added. Business Information System Engineering. 2015; 57: 221-224.

[9] Satpute S, Deora BS. Improving performance of internet of things by using local IoT controller unit. International Conference on Green Computing and Internet of Things (ICGCIoT). 2015; 1328-1330.

[10] Miraz MH, Ali M, Excell PS, Picking R. A review on internet of things (IoT), internet of everthing (IoE) and internet of nano things (IoNT). Internet Technologies and Applications. 2015; 219-224.

[11] Atzori L, Lera A, Morabito G. Understanding the Internet of Things: Definition, potentials, and societal role of a fast evolving paradigm. Ad Hoc Networks, Elsevier. 2016.

[12] Lueth KL. IoT basics: Getting started with the internet of things [Internet]. 2015.

[13] Alliance for Internet of Things Innovation. Identifiers in Internet of Things (IoT). AIOTI Version 1.0. 2018; 1-34.

[14] Kumar S, Tiwari P, Zymbler M. Internet of Things is a revolutionary approach for future technology enhancement: a review. Journal of Big Data. 2019; 1-21.

[15] Hassan R, Qamar F, Hasan MK, Aman AHM, Ahmed AS. Internet of Things and Its Applications: A Comprehensive Survey. Symmetry. 2020; 12: 1-29. 
[16] Internet Society. The Internet of Things (IoT): An Overview Understanding the Issues and Challenges of a More Connected World [Internet]. 2016.

[17] Ranger S. What is the IoT? Everything you need to know about the Internet of Things right now, ZDNET [Internet]. 2020.

[18] Heater B. Echo auto brings alexa to cars [Internet]. 2018.

[19] FIXD Automotive. Why every driver should own a FIXD sensor [Internet]. 2021.

[20] Apple Inc. Car keys and CarPlay: A smarter ride from start to finish [Internet]. 2021.

[21] Charlton A. Android auto: Google's head unit for cars [Internet]. 2018.

[22] Heflin M. The nervous system of the IoT [Internet]. 2016.

[23] Malik A, Magar TA, Verma H, Singh M, Sagar PA. Detailed Study of an Internet of Things (IoT). International Journal of Scientific \& Technology Research. 2019; 8.

[24] Press G. A very short history of the internet of things [Internet]. 2014. 